\title{
L'hydrométéorologie Opérationnelle à Electricité de France
}

\author{
D. Duband
}

EDF

\section{I INTRODUCTION}

L'hydrologie est la science des quantités d'eau, en circulation ou en réserve, sur les continents. L'eau est un élément essentiel dans les activités humaines: l'alimentation en eau potable des hommes et des animaux, l'irrigation et le drainage dans l'agriculture, la pêche, la production d'énergie, les industries de transformation, l'évacuation des eaux usées et pluviales, les loisirs en rivière ou sur plan d'eau, etc...

Outre son importance dans la gestion des ressources, non seulement en période de sécheresse mais aussi face aux usages multiples en eau, l'hydrologie permet de se prémunir contre les effets destructeurs de l'eau que sont les crues, les inondations, l'érosion. Elle est donc fondamentale pour l'aménagement des vallées où sont concentrées les villes, les industries, les voies de transport et l'agriculture. Elle prend toute son importance également dans les processus concourant à la qualité de l'eau (hydrobiologie, poissons, ...) des hydrosystèmes.

L'eau qui tombe sous forme de pluie ou de neige sur les continents est en majeure partie réévaporée par le sol et la végétation : l'écoulement qui apparaît dans les cours d'eau ne représente qu'une fraction de la précipitation $(30 \%$ en zone tempérée, plus de $60 \%$ en zone équatoriale ou tropicale, moins de $15 \%$ en zone semi-aride et aride), après qu'une partie de celle-ci se soit infiltrée dans les nappes phréatiques.

Dans le bilan hydrologique, l'écoulement de surface est le seul terme qui soit mesurable en vraie grandeur. Tous les autres termes - précipitations - enneigement évaporation - condensation - infiltration - échanges souterrains (nappes phréatiques) sont connus seulement par des mesures ponctuelles, utilisables comme des indices plus ou moins représentatifs des phénomènes spatiaux.

Aux difficultés de mesure dues à la variabilité dans l'espace s'ajoutent l'irrégularité dans le temps des phénomènes, les précipitations par exemple les déphasages dans les processus de transfert, et surtout l'aléa des conditions météorologiques futures : il apparaît alors plus opportun de parler de l'hydrométéorologie.

De ce fait, le recours aux méthodes physico-statistiques s'impose en hydrométéorologie opérationnelle autant et même plus que dans les autres sciences géophysiques. Les équations qui servent à évaluer et prévoir, lorsque c'est possible, les débits et les apports des rivières tant en valeurs courantes qu'extrêmes ne sont jamais des bilans déterministes mais des liaisons relatives ou corrélatives assorties d'une incertitude résiduelle qui doit être chiffrée.
Pour de nombreuses applications, il est nécessaire de décrire numériquement la variabilité spatio-temporelle et cela n'est possible qu'en s'appuyant sur des séries historiques de mesures fines (minute, heure, jour) effectuées pendant de nombreuses années. Les réseaux de mesure hydrologiques et climatologiques constituent un investissement à long terme et un capital irremplaçable du patrimoine de chaque pays pour la conception et l'exploitation des aménagements de cours d'eau, la planification agricole urbaine et industrielle et la lutte contre les risques naturels et technologiques (crues, inondations, sécheresse, pollutions, ...).

La Division Technique Générale (Service Ressources en Eau) a développé une activité d'ingénierie et un savoirfaire dans l'entreprise publique Electricité de France, pour contribuer à résoudre au mieux techniquement et économiquement la satisfaction globale des besoins en eau actuels et futurs d'un bassin en France mais aussi en Afrique, Asie, Amérique du Sud...

Ces besoins dépendent de l'évolution des multiples usages de l'eau énergétique - industriel - agricole - eau potable - loisirs - pêche, parfois antagonistes pour les activités humaines, associés à une protection équilibrée de l'environnement aquatique (vie piscicole, zones végétales, salubrité...) et nécessitent des mesures de terrain, des études, des prévisions, des calculs économiques réalisés à la demande.

Cette activité intégrée peut être segmentée en cinq domaines d'intervention :

- exploitation des réseaux de mesure permanents,

- calcul des prévisions en temps réel,

- prédétermination des risques d'événements extrêmes,

- maîtrise et prévention des évolutions de l'environnement,

- études économiques et recommandations pour une meilleure exploitation partagée des ressources en eau.

Sur le terrain, la DTG gère des réseaux de mesure pérennes et temporaires à partir de capteurs avec systèmes d'acquisition installés dans les stations, en temps réel (par téléphone, satellite, radio) et en différé (mémoire statique, diagramme...).

Ces données enrichissent les séries de mesures déjà archivées dans les banques de données historiques et utilisées dans les modèles économiques, les modèles physicostatistiques de prévision et de calcul de risques naturels, les modèles de qualité de l'eau. 
II — CRÉATION ET EXPLOITATION DE RÉSEAUX DE MESURE PÉRENNES/ BANQUES DE DONNÉES

Cette activité, qui va contribuer à constituer le capital de données, distingue une suite d'opérations indissociables:

- le choix des sites, des méthodes et moyens de mesure, - l'optimisation de l'échantillonnage spatial et temporel de l'information attendue,

- la réalisation des mesures de paramètres de quantités et de qualité de l'eau,

- l'interrogation en temps réel des capteurs (acquisition, transmission),

- la critique et la validation des mesures,

- la diffusion de l'information,

- l'alimentation de banques de données qui vont enrichir les connaissances par leur dimension historique et chronologique.

EDF a l'expérience d'installation et maintenance de près de 1200 stations de mesure dont près de la moitié sont télémesurées (fig. 1).

Les précipitations sont mesurées à partir du seau totalisateur ou du pluviomètre enregistreur avec ou sans chauffage, à augets basculants. L'équivalent en eau et l'épaisseur du manteau neigeux sont mesurés soit par sondage manuel soit par des appareils automatiques autonomes, les télénivomètres. La température de l'air est mesurée par thermographe ou sonde.

On déduit le débit des cours d'eau du niveau enregistré en continu (par jauge de contrainte, ultrason, ...) par une loi hauteur - débit établie d'après des jaugeages effectués soit par mesure mécanique (moulinet) ou chimique (dilution d'un traceur) -700 à 1000 jaugeages/an.

La température de l'eau est mesurée ponctuellement, avec vérifications de sa représentativité par des profils thermiques périodiques dans la section de rivière.

D'autres paramètres physico-chimiques de qualité d'eau sont également mesurés en continu comme l'oxygène dissous, la conductivité, le $\mathrm{pH}$, la turbidité, l'ammoniac...

La collecte, le traitement informatique, le contrôle, la critique, l'archivage et la publication des résultats de mesure sont réalisés à l'aide de logiciels et moyens informatiques souples et adaptés, sans pour autant masquer la réalité physique.

\section{III $\square$ CALCUL ET ÉLABORATION DES PRÉ- VISIONS EN TEMPS RÉEL}

L'hydrologie, ce n'est pas seulement la mesure de l'état actuel des paramètres hydrométriques, pluviométriques, nivométriques, thermiques, c'est aussi l'appréhension an-

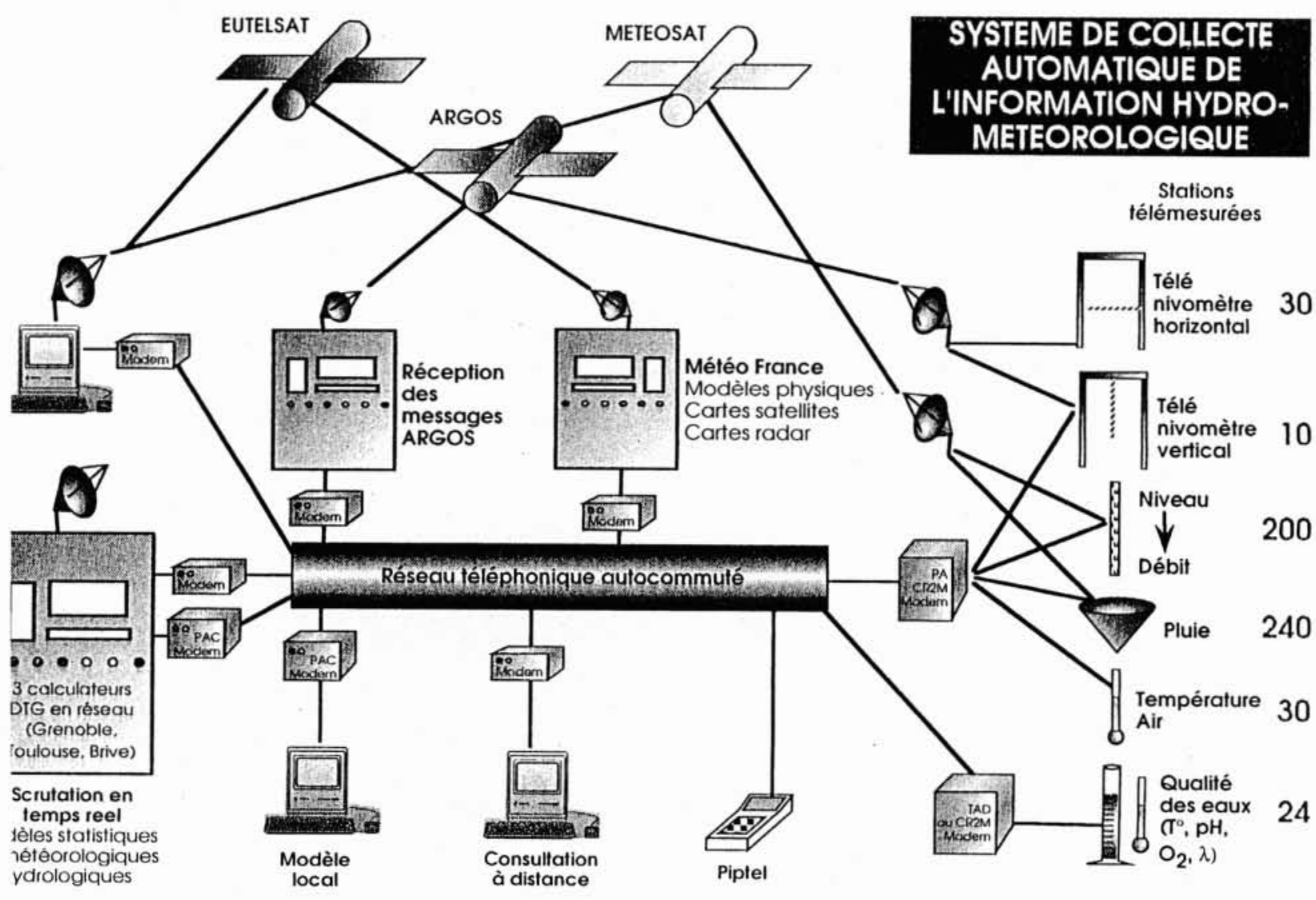

1. Système de collecte automatique de l'information hydrométéorologique. 
ticipée de leur évolution dans les prochaines heures prochains jours ou même prochains mois dès la fin de 1'hiver. Les procédures numériques mises au point vont donc de la prévision des apports de fusion nivale, à la prévision des crues, en passant par la prévision des étiages (fig. 5).

On ne peut dissocier l'hydrologie opérationnelle de la météorologie dynamique, de même qu'on ne peut séparer I'hydrologie classique de la climatologie. L'expérience quotidienne d'association de la situation météorologique observée et prévue, à la situation hydrologique de terrain, pendant plus de vingt ans, nous permet de jouer le rôle d'interface entre Météorologie et Hydrologie. Grâce aux cartes synoptiques et données numériques observées et prévues 1 à 6 jours à l'avance, on établit des prévisions d'évolution en tendance de la précipitation à l'hydraulicité, de la température de l'air pour chaque bassin versant et région par sélection de situations météorologiques historiques, stockées en mémoire depuis 40 ans (fig. 2).
Les modèles utilisés pour la prévision de débits à court terme ont été choisis volontairement simples, robustes, économiques pour être efficaces en application opérationnelle en temps réel, et suffisamment proches du processus physique.

Sur les bassins versants où la transformation de la précipitation brute $P$ reçue, en pluie efficace $P E$ et ruissellement direct $Q$, est prépondérante par rapport à la propagation, on calcule les variations prévues de débits futurs d'après un modèle linéaire du type :

$$
\Delta Q_{h}=\Sigma a_{i} P E_{h-i+1} .
$$

La pluie efficace d'un intervalle de temps passé est estimée d'après un modèle de production Pluie brute-Pluie efficace, du type :

$$
P E=\frac{P^{2}}{P+b}
$$

(d'autres modèles sont testés, TOP, réservoirs).

\section{La DTG, interlocuteur opérationnel des \\ Centres de Décision EDF, en interface avec Météo France.}
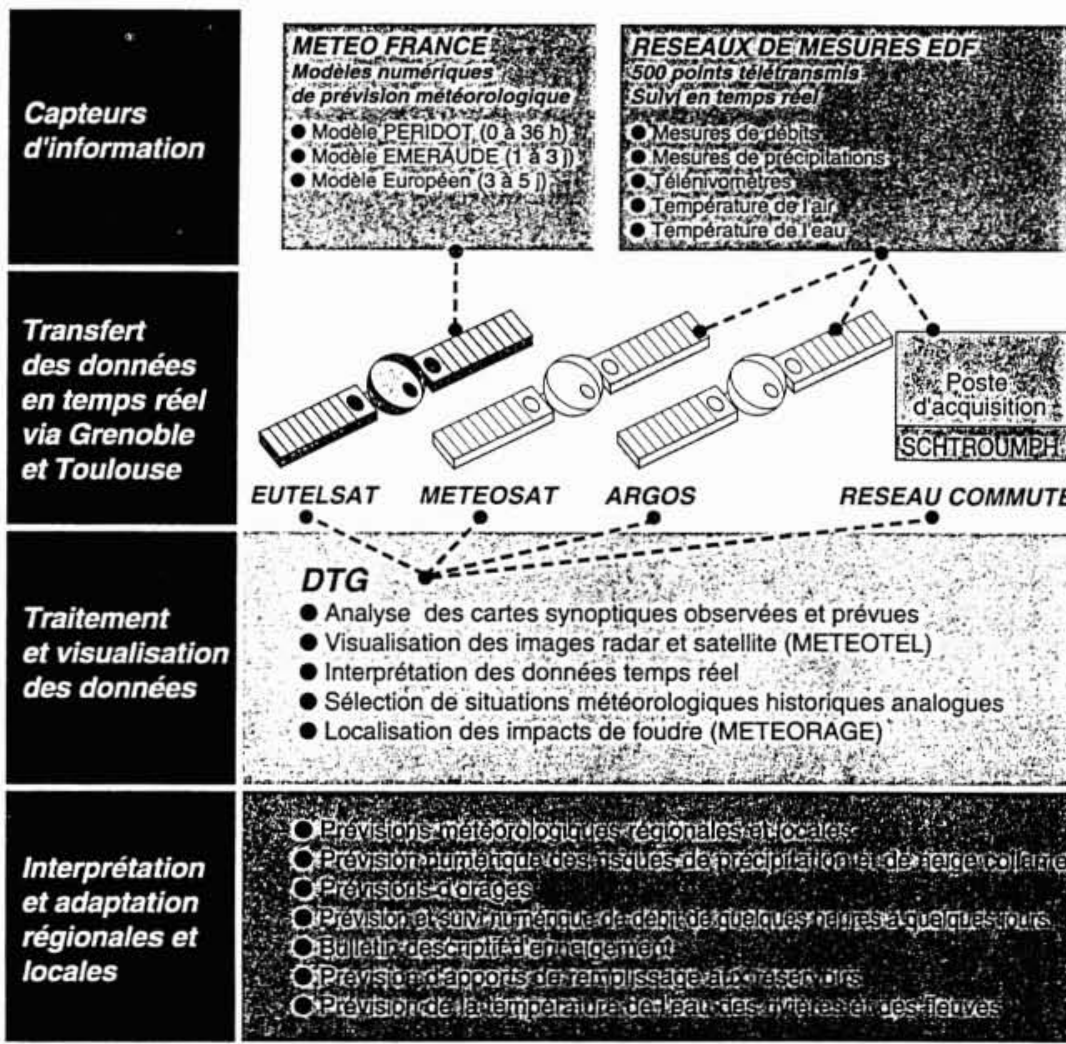

- Analyse des cartes synoptiques observées et prévues

- Visualisation des images radar et satellite (METEOTEL)

- Interprétation des données temps réel

- Localisation des impacts de foudre (METEORAGE)

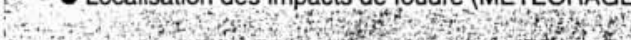

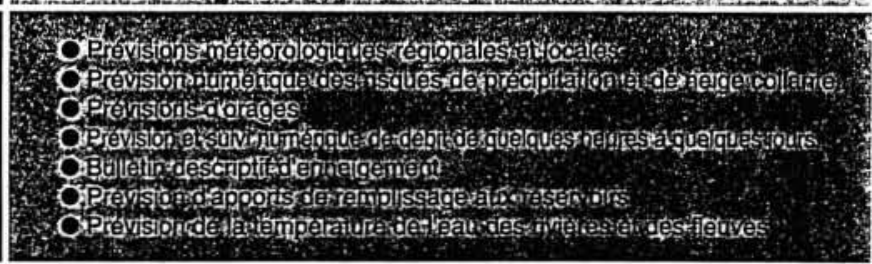

Diffusion temps réel des données et des prévisions aux Centres de Décision EDF

2. Prévisions et suivi hydrométéorologique à EDF. 
EXEMPLE DE PREVISION DE DEBITS DE LA BOURNE A PONT-DE-MANNE AU PAS DE TEMPS DE DEUX HEURES, D'APRES LES PRECIPITATIONS OBSERVEES PENDANT L'AVERSE DES 5 ET 6 FEVRIER 1979

Le calcul de la prévision des débits au pas de temps de 2 heures est effectué pour une cinquantaine d'heures futures, en réduisant la pluie observée en pluie nette disponible pour le ruissellement direct. On fait l'hypothèse que les pluies futures seront nulles, la prévision est réactualisée toutes les 2 heures.
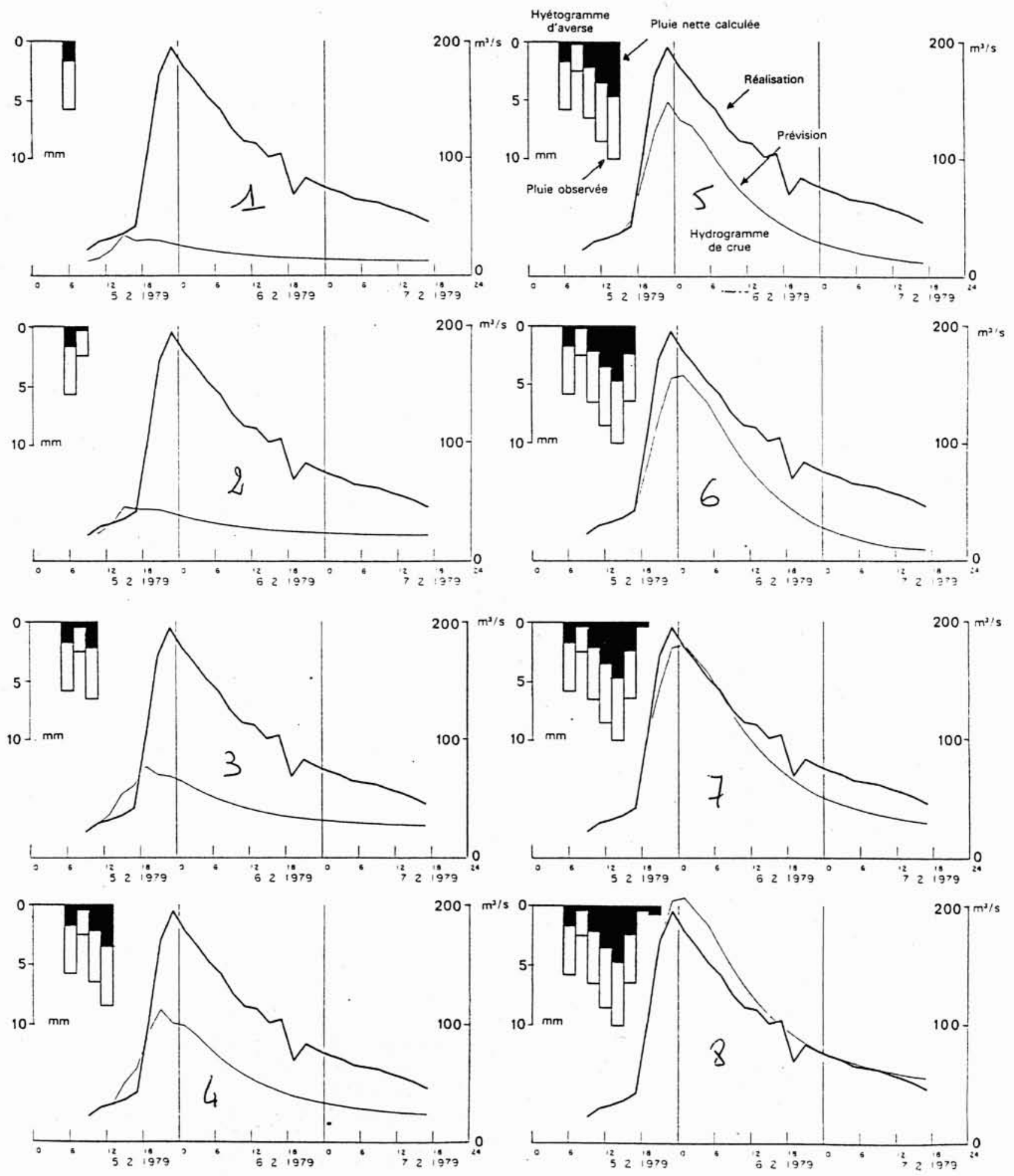

3. Exemple de prévision de débits de la Bourne à Pont-de-Manne au pas de temps de deux heures, d'après les précipitations observées pendant l'averse des 5 et 6 février 1979. 
Les coefficients « $a$ » sont ceux de la Différence Première de la Fonction de transfert moyenne du bassin, ou hydrogramme unitaire. Cette approche permet de recaler régulièrement les prévisions de débit pour les prochaines heures sur le dernier débit connu, l'approche directe $Q_{h}=\Sigma A i P E_{h-i+1}$ constituant un modèle dégradé (fig. 3 ).

Nous avons mis au point, à la fin des années 70 , une méthode de calage originale des paramètres de ces modèles d'après des échantillons historiques de mesures des couples averse-crue, et appliquée à une trentaine de bassins.

Lorsque la propagation devient prépondérante on utilise des relations multilinéaires entre variations de débits témoins (d'affluents) amont et aval étalonnés sur des mesures historiques :

$$
\Delta Q_{h}=\Sigma b i \Delta Q_{1, h-i}+\Sigma c i Q_{2, h-i}+\cdots \cdot
$$

La solution retenue par EDF consiste à intégrer le logiciel d'acquisition des données hydrologiques et les modèles de prévision sur des micro-ordinateurs reliés à des calculateurs centraux.

Quant à la prévision des apports dans une cinquantaine de réservoirs de haute montagne pendant le printemps et l'été, qui est l'une des bases de l'activité hydrologique à EDF pour la gestion du productible Lac et les achats de combustibles, elle s'est affinée au fil des ans par une meilleure connaissance de l'enneigement, venant compléter la mesure des précipitations d'hiver et l'estimation de l'état des réserves hydriques du sol, qui constituent les éléments de calcul des prévisions, réactualisées chaque mois ou quinzaine de janvier à mai (fig. 4).

\section{IV — PRÉDÉTERMINATION DES RISQUES D'ÉVÉNEMENTS NATURELS EXTRÊ- MES -}

La connaissance préalable de la probabilité d'événements hydroclimatologiques rares et catastrophiques (crues, pluies, précipitations neigeuses, neige collante et lourde, étiages, canicule, vague de froid) s'appuie sur l'analyse fréquentielle des données historiques ainsi que des hypothèses physiques simples pour guider non seulement la stratégie de la prévention mais aussi celle de la prévision.

Afin d'optimiser le dimensionnement des ouvrages d'évacuation assurant la protection des grands barrages, il convient de déterminer l'intensité des plus fortes crues susceptibles de se présenter.

Deux approches susceptibles de se compléter sont actuellement utilisées pour cette évaluation :

- l'une consiste à déterminer la « crue maximale probable " sans affectation de probabilité, correspondant à la crue de sécurité,

- l'autre, pour atteindre cette crue de sécurité, consiste en la prise en compte d'une crue de projet de probabilité donnée $\left(10^{-3}\right.$ ou $\left.10^{-4}\right)$ et d'une revanche. La méthode du «gradex» (gradient de la fréquence exponentielle des valeurs extrêmes de précipitation et de débit), est proposée par le Comité Français des Grands Barrages, dans cette logique.

Le gradex des pluies est une valeur caractéristique du régime des pluies, qui varie selon la saison et le lieu géographique. En calculant une estimation fiable du gradex de la saison où les risques de pluie sont les plus forts, l'on
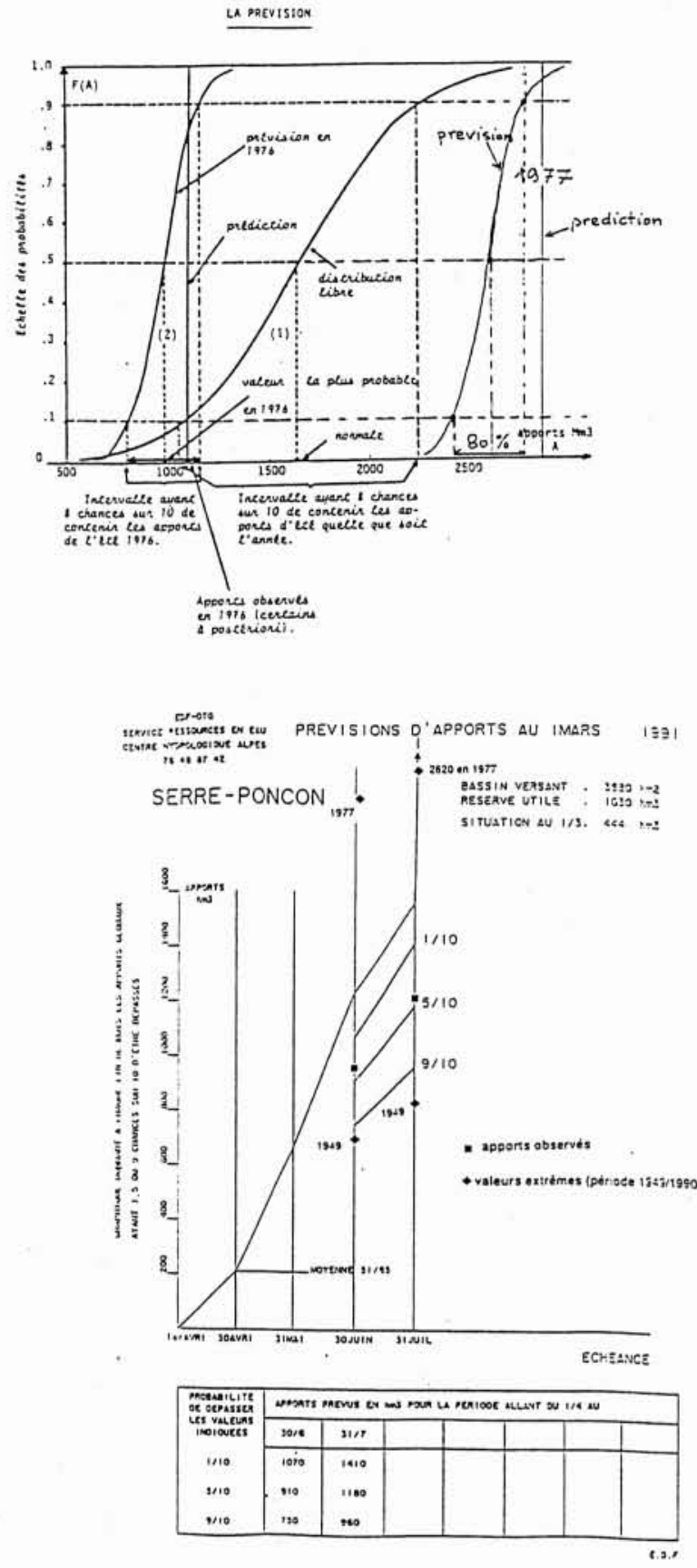

\section{Prévision des apports à Serre-Ponçon.}

en déduit celui des risques de pluies extrêmes annuelles, avec seulement quelques dizaines d'années d'observations pluviométriques comme base de calcul. Il ne faut pas prendre en compte la précipitation la plus intense observée, mais la potentialité que présente un site, un bassin donné à recevoir des intensités de pluie d'une certaine échelle.

Cette méthode est une approche tout à la fois physique, statistique et climatologique.

La donnée capitale est que la capacité de rétention d'un bassin tend à devenir limitée pour de fortes averses, car au-delà d'une certaine hauteur de précipitation le déficit d'écoulement cesse de croître, selon l'état de saturation préalable du sol. 


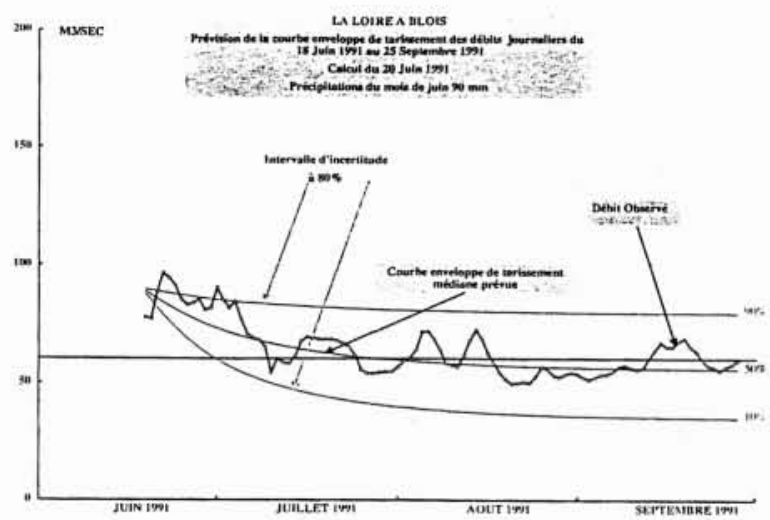

Prévision de tarissement des débits de la Loire à Blois. Prevision du 8 juin 1992.

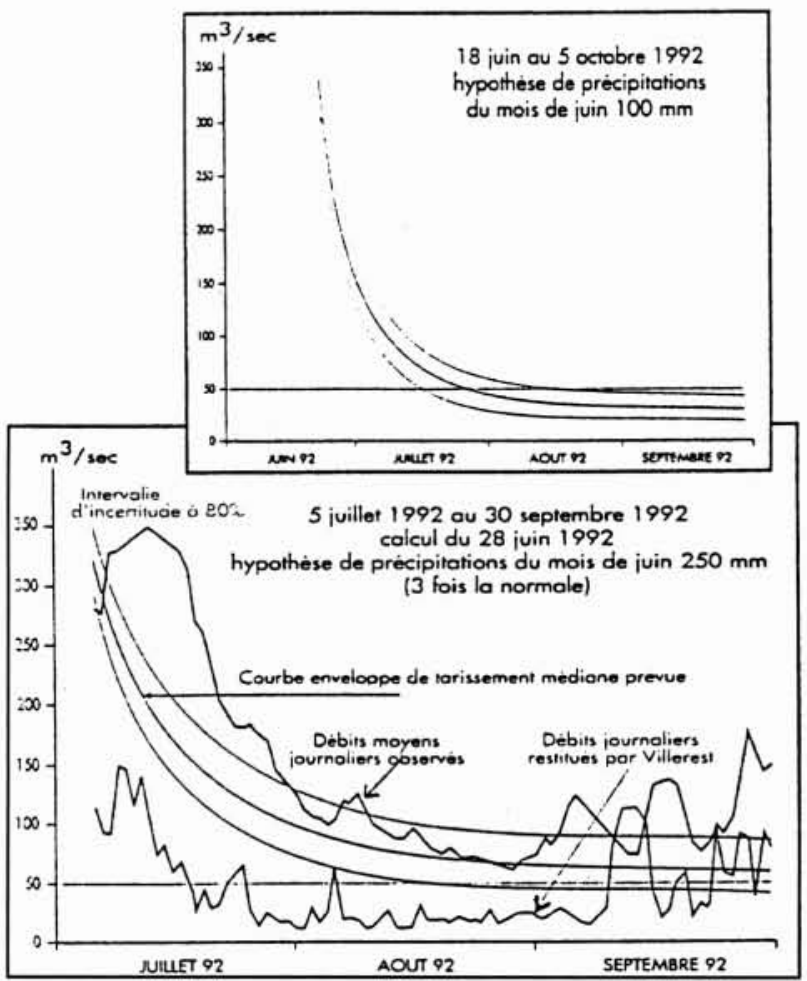

Prévision de tarissement des débits de la Loire à Blois. Prévision du 28 juin 1992.

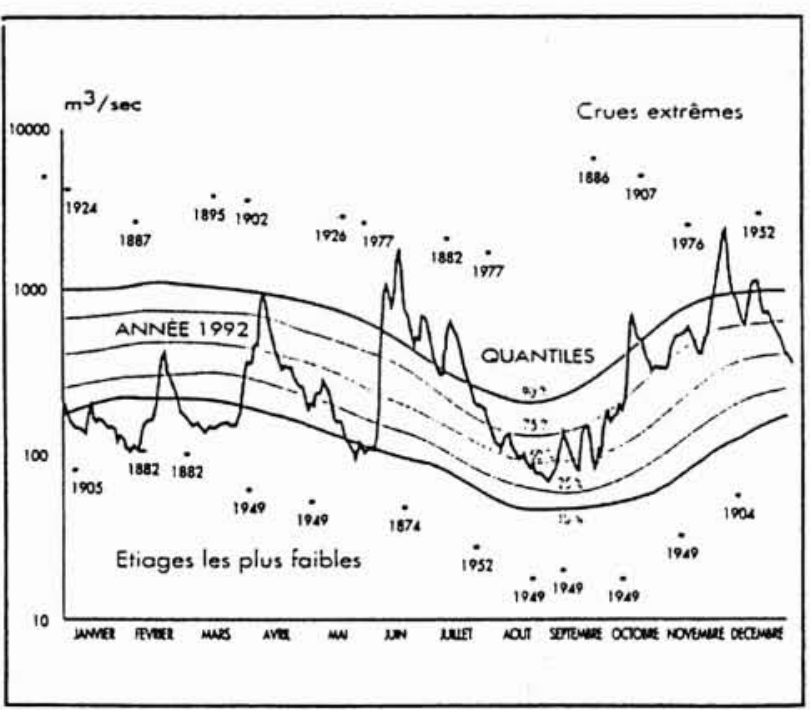

Débits moyens journaliers de la Loire à Blois. Situation de l'année 1992 par rapport oux quantiles ètablis d'aprés les chroniques de débit moyen joumalier sur la période 1863-1977.

5. La Loire à Blois : prévision de la courbe enveloppe de tarissement des débits journaliers du 18 juin 1991 au 25 septembre 1991.

Le calcul comporte deux variables aléatoires : l'une à champ borné (capacité d'absorption du sol), l'autre non bornée (pluies extrêmes) qui devient prédominante. C'est pourquoi les comportements asymptotiques des fréquences des pluies et des débits sont sinon identiques, du moins du même type, et se correspondent à un décalage près. Cette méthode, qui intègre le traitement des données pluviométriques sur de longues périodes, est donc une bonne optimisation entre la crue historique et la crue maximale théoriquement possible.
Des études de ce type ont été réalisées sur 250 bassins versants en France et ailleurs dans le monde, avec le logiciel GRADEX (fig. 6 et 7).

\section{MAÎTRISE ET PRÉVISION DES ÉVO- LUTIONS DE L'ENVIRONNEMENT}

Les aménagements en rivières entraînent, dans certains cas, une modification de la vie aquatique et de la qualité de l'eau. 


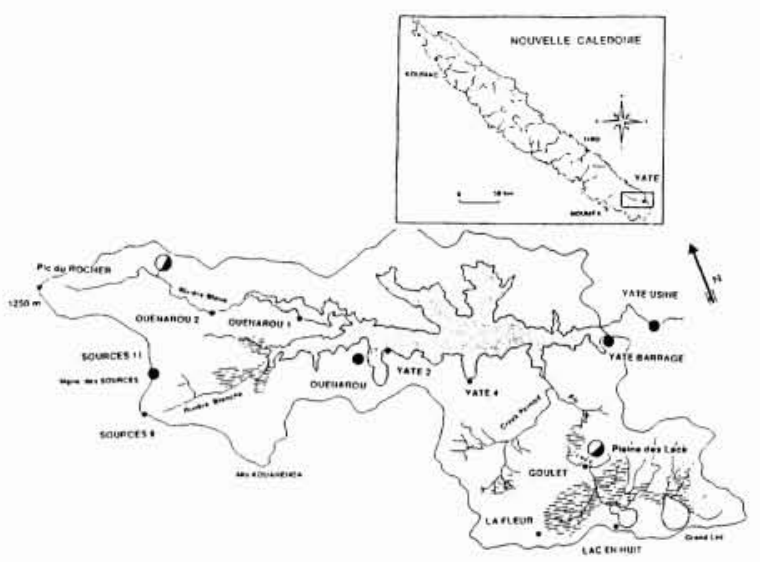

6. Application de la méthode Gradex en NouvelleCalédonie.

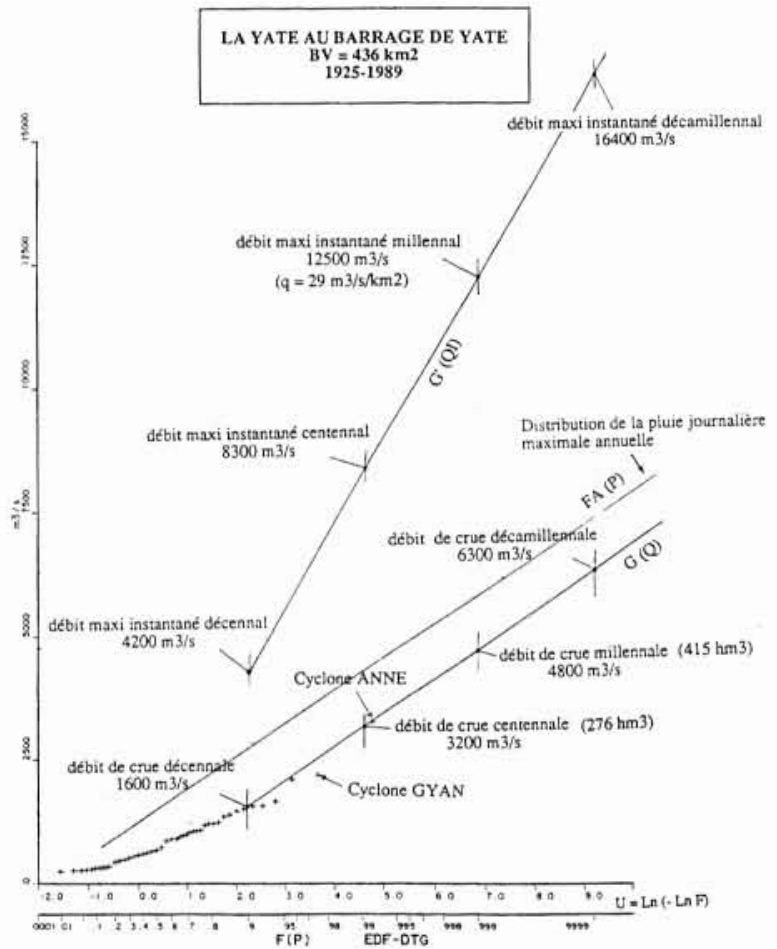

7. Débit de la Yate au barrage de Yate.

C'est pourquoi, il est indispensable d'étudier l'évolution et les nouveaux besoins de l'environnement pour instaurer une gestion équilibrée des milieux aquatiques en s'appuyant sur le savoir-faire et l'expertise d'EDF pour :

- les migrations piscicoles,

- les topographies sous-marines des rivières et des retenues (Bathymétrie),

- les vidanges de barrages, pour en limiter les impacts,
- les matières en suspension (turbidité et micropolluants),

- la dynamique et la pollution des sédiments.

- la situation et l'évolution des nappes phréatiques,

- la réalisation de tableaux de bord de bassin pour le suivi et la gestion décisionnelle.

\section{VI ÉTUDES ET RECOMMANDATIONS POUR UNE MEILLEURE EXPLOITA- TION PARTAGÉE DES RESSOURCES EN EAU}

Le service Ressources en Eau étudie et chiffre la rentabilité de la meilleure répartition de l'eau entre ses différents usages (énergie, environnement, agriculture, eau potable, tourisme...) à l'aide du logiciel PARSIFAL en :

- simulant une exploitation hydrologique et hydraulique optimale par modélisation :

- calculant la valeur de l'eau en fonction de la demande et de l'usage :

— fournissant des scénarii pour la gestion prévisionnelle des grands stocks d'eau ;

- calculant l'actif d'une usine hydroélectrique :

- calculant le coût de diverses contraintes environnementales, touristiques.

\section{Références}

[1] Prévision et télésurveillance hydrométéorologique à EDF. P. Tourasse, EDF Division Technique Générale. Houille Blanche $\mathrm{n}^{\circ} 2,1991$.

[2] Unit hydrograph revisited : an alternate iterative approach to $U H$ and effective precipitation identification. D. Duband, Ch. Obled, J.Y. Rodriguez. Journal of Hydrology 150 (1993), 115-149.

[3] 40 years of snow cover measurement in France: network evaluation and forecast interest. Western Snow Conference, Jackson Hole, Wyoming, USA, April 1992

[4] Les crues de projet des barrages: Méthodes du Gradex. Bulletin du Comité Français des Grands Barrages, FR COLD NEWS. $18^{\mathrm{c}}$ Congrès CIGB/COLD, novembre 1994, Durban, Afrique du Sud.

[5] Gestion des vidanges de réservoirs. Mesures prises pour préserver l'environnement et retour d'expérience sur plus de 60 vidanges. A. Poirel, E. Vindimian, J. Garric. $18^{\mathrm{e}}$ Congrès des Grands Barrages, novembre 1994, Durban, Afrique du Sud.

[6] Gestion piscicole lors des vidanges de barrage. A. Poirel et C. Lallement. Entretiens Jacques Cartier, Chambéry, décembre 94 . France.

[7] Quelle politique en matière de capteurs et d'instrumentation. C. Lallment. H20, 1993, Alpexo, Grenoble, France.

[8] A management Method and associate software for Hydroelectric Systems with one or two reservoirs. J.M. Bourgueil. Symposium Computational Mechanics, juillet 1992, Valencia, Espagne. 